\title{
Understanding the structural characteristics of compstatin by conformational space annealing
}

\author{
Mee Kyung Song, Seung-Yeon Kim, Jooyoung Lee* \\ School of Computational Sciences, Korea Institute for Advanced Study, 207-43 Cheongnyangni 2-dong, Dongdaemun-gu, Seoul 130-722, Korea
}

Received 30 June 2004; received in revised form 1 November 2004; accepted 10 December 2004

Available online 11 January 2005

\begin{abstract}
The structural characteristics of the 13-residue compstatin molecule are investigated using the conformational space annealing (CSA) method with CHARMM force field and the GBSA continuum solvent model. In order to sample conformations in the energy range of the minimized NMR structures, we have used the stopping criterion to the CSA search when a conformation whose energy is less than -490 $\mathrm{kcal} / \mathrm{mol}$ is found. With this stopping criterion, a great variety of conformations are generated around experimentally known structures. Twenty independent CSA runs starting from random states find 1000 representative conformations in the energy landscape of the compstatin, which are classified into thirty-one structural families. The majority of the conformations (94.4\%) are in the coil state. Other conformers containing a 3 -helix, a $\pi$-helix, a $\beta$-hairpin, and an $\alpha$-helix are also found.
\end{abstract}

(C) 2004 Elsevier B.V. All rights reserved.

Keywords: Compstatin; Complement inhibitor peptide; Conformational space annealing; Energy landscape; $\beta$-turn

\section{Introduction}

The complement systems play an essential role in the defense of a host organism against infectious agents and the inflammatory process [1]. They cause lysing or bursting of cells and they send signals to phagocytes that a cell needs to be removed. The complement systems can be activated by three different pathways: the classical, alternative, or lectin pathways [2]. Their activation is essential for the development of normal inflammatory responses against foreign pathogens. However, inappropriate complement activation leads to the host cell damage [3] and is implicated in a variety of pathological conditions including autoimmune disease, stroke, Alzheimer's disease, adult respiratory distress syndrome, heart attack, and burn injuries [4-10]. Thus, there is a clear need for specific complement inhibitors with therapeutic application in a wide variety of conditions.

Compstatin is a novel complement inhibitor with potential to be developed into a therapeutic agent. Compstatin inhibits

\footnotetext{
* Corresponding author. Tel.: +82 2958 3731; fax: +82 29583786 .

E-mail address: jlee@kias.re.kr (J. Lee).
}

the complement activation by binding specifically to the third complement component $\mathrm{C} 3$ that plays a central role in activation of the complement systems. Because of its high activity, no significant side effects, low toxicity and structural simplicity, compstatin is a promising candidate for drug development [11-13]. The three-dimensional structures of compstatin in solution were investigated by the NMR spectroscopy [14]. The amino acid sequence of compstatin is $\mathrm{Ile}^{1}-\mathrm{Cys}^{2}-\mathrm{Val}^{3}-\mathrm{Val}^{4}-\mathrm{Gln}^{5}-\mathrm{Asp}^{6}-\mathrm{Trp}^{7}-\mathrm{Gly}^{8}{ }^{8} \mathrm{His}^{9}-\mathrm{His}^{10}$ $\mathrm{Arg}^{11}-\mathrm{Cys}^{12}-\mathrm{Thr}^{13}$, where $\mathrm{Cys}^{2}$ and Cys ${ }^{12}$ are bridged by a disulfide bond and $\mathrm{Gln}^{5}-\mathrm{Asp}^{6}-\mathrm{Trp}^{7}-\mathrm{Gly}^{8}$ forms a $\beta$-turn. The disulfide bridge, the four residues of the $\beta$-turn, and $\mathrm{Val}^{3}$ are known to be essential for the inhibitory activity [11-13].

Recently, the energy landscape of proteins has been extensively investigated to understand the nature of protein folding $[15,16]$. However, for real proteins, all-atom representations that include all pairwise interactions and solvation effects fail to characterize the energy landscape, since they require astronomical amounts of CPU time. Mallik et al. [17] has investigated the energy landscape of compstatin using 1ns molecular dynamics (MD) simulations starting from the 21 NMR structures [14], using a CHARMM force field with a 
continuum solvent model. They found three kinds of conformers, coil with a $\beta$-turn, $\beta$-hairpin with a $\beta$-turn, and an $\alpha$-helix, at the end of their MD simulation. However, in their MD study, only small perturbation from the NMR structures of compstatin is allowed, and consequently only a small fraction of the conformational space of compstatin has been investigated.

In this study, we investigate the energy landscape of compstatin starting from unfolded states, using the conformational space annealing (CSA) method $[18,19]$ and a CHARMM force field with a continuum solvent model. The structural characteristics of the various conformers for compstatin are identified.

\section{Methods}

\subsection{Potential energy function}

The calculations were carried out using the CHARMM potential energy function [20]. Standard CHARMM energy terms and the Generalized Born (GB) implicit solvation model (GBSA: GB with Solvent Accessible Surface Area) in the TINKER package (http://dasher.wustl.edu/tinker/) were used. No long-distance truncation was applied for nonbonded interactions. The toph19 and param 19 were used as the topology and parameter file of the CHARMM, respectively. A charged $\mathrm{N}$-terminus was used with the normal patch of $\mathrm{NH}_{3}^{+}$, and $\mathrm{C}$-terminal residue was blocked with $\mathrm{NH}_{2}$ to account for the experimental C-terminal blocking [14]. The all-hydrogen protein topology and parameters, top_all22_prot.inp and par_all22_prot.inp, respectively, were adopted for the amidated $\mathrm{C}$-terminus. A harmonic potential was used in order to maintain the disulfide bond between Cys2 and Cys12.

\subsection{Conformational space annealing (CSA)}

The energy landscape of compstatin can be investigated by CSA method $[18,19]$. In CSA, we consider only the phase space of local minima, that is, all conformations are energyminimized by a local minimizer. We assume that most of the phase space of local minima can be covered by a finite number of large spheres with radius $D_{\text {cut }}$, which are centered on randomly chosen minima (bank). Each of the bank conformations is supposed to represent all local minima contained in the sphere centered on it. To improve a bank conformation $A$, we first select $A$ as a seed. We perturb $A$ and subsequently energy-minimize it to generate a trial conformation $\alpha$. Since $\alpha$ originates from $A$ by small perturbation, it is likely that $\alpha$ is contained in the sphere centered on $A$. If the energy of $\alpha$ is lower than that of $A, \alpha$ replaces $A$ and the center of the sphere moves from $A$ to $\alpha$. If it happens that $\alpha$ belongs to a different sphere centered on $B, \alpha$ can replace $B$ in a similar manner. When $\alpha$ is outside of all existing spheres, a new sphere centered on $\alpha$ is generated. In this case, to keep the total number of spheres fixed, we remove the sphere represented by the highest-energy conformation. Obviously, the former two cases are more likely to happen when spheres are large, and the latter when spheres are small. Consequently, larger value of $D_{\text {cut }}$ produces more diverse sampling, whereas smaller value results in quicker search of low-energy conformations at the expense of getting trapped in a basin probably far away from the global minimum. Therefore, for efficient sampling of the phase space, it is necessary to maintain the diversity of sampling in the early stages and then gradually shift the emphasis toward obtaining low energy conformations, which is realized, in CSA, by slowly reducing the value of $D_{\text {cut }}$. Finally, representative conformations in the energy landscape of a protein can be obtained by CSA. In this work, twenty independent CSA runs for compstatin are carried out, starting from a bank of fifty conformations. Each run has fifty conformations in the final bank, and thus, a total of 1000 conformations are analyzed.

\subsection{Stopping criterion for practical application of CSA}

For the practical application of CSA to compstatin, we have used the following stopping criterion. When 21 NMR compstatin structures [14] are minimized using the CHARMM potential function with the GBSA continuum solvent model as described in Section 2.1, their energies range between -482.84 and $-453.71 \mathrm{kcal} / \mathrm{mol}$ (see Section 3 and Table 2). In order to sample conformations around this energy range, we have terminated the CSA search when a conformation whose energy is less than $-490 \mathrm{kcal} / \mathrm{mol}$ is found. If we do not use this stopping criterion and continue the CSA search, very low energy conformations with energy $\sim-520 \mathrm{kcal} / \mathrm{mol}$ are obtained in the bank. Fig. 1 shows the backbone root-mean-square-deviation (rmsd) as a function of energy for all 36250 distinct conformations without utilizing the above mentioned stopping criterion. The

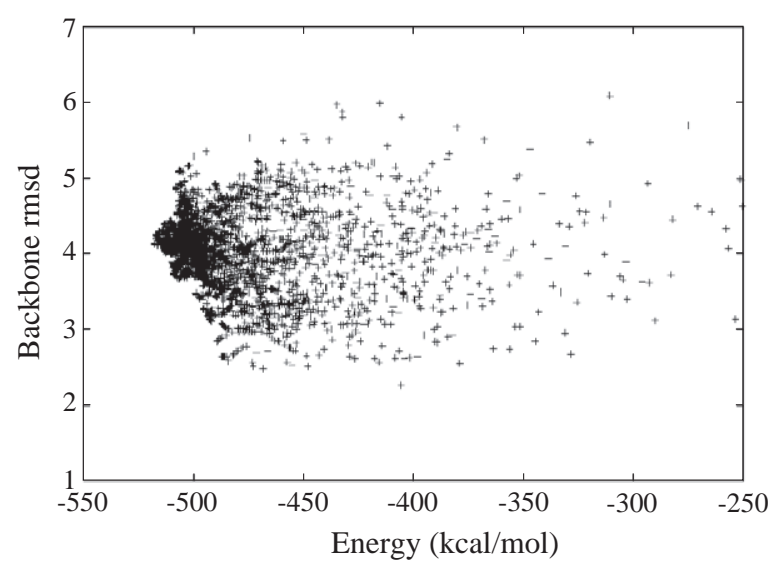

Fig. 1. Distribution of backbone rmsd values as a function of energy for all 36250 conformations generated during a CSA run without the stopping criterion. The energies of the final bank conformations range between -501 and $-518 \mathrm{kcal} / \mathrm{mol}$. These low energy conformations are almost all in coil states without $\beta$-turns. 
backbone rmsd is calculated from the minimized structure of the average compstatin structure. The average structure of compstatin is obtained by averaging the coordinates of 21 NMR structures (more details on this are given in Section $3)$. Energies of the final conformations are as low as about $-520 \mathrm{kcal} / \mathrm{mol}$. These low energy conformations are almost all in coil states without $\beta$-turns. It should be noted that all 21 NMR structures are coils with a $\beta$-turn. The deficiency of the force field may be a reason why the lowest-energy conformations appear to be inconsistent with the NMR data. Another reason may be that the ensemble obtained within the restricted energy range has much higher configurational entropy than that with the lowest potential energy. Therefore, this particular stopping criterion was utilized to sample conformations specifically in the energy range of the minimized NMR structures, and to obtain possible compstatin structures that can arise from the energetics of the CHARMM potential with the GBSA continuum solvent model. With this stopping criterion, a great variety of conformations are generated around experimentally known structures. The goal of this work is to investigate and analyze these conformations in order to provide relevant relationship between them and the experimental structures.

\section{Results and discussion}

The solution structures of compstatin (PDB ID: 1a1p) are obtained using two-dimensional NMR techniques [14]. The conformations of the 21 NMR structures are represented as coil $/ \alpha_{R}-\alpha_{R}$. Here, the secondary structure of the whole compstatin and the type of the $\beta$-turn at residue numbers 5-8 are denoted before and after the slash, respectively. The three-state secondary structure classification of coil, strand, and helix is determined by the DSSP algorithm [21] implemented in the program MOLMOL [22]. The $\alpha_{R}-\alpha_{R}$ represents a type of $\beta$-turn that is determined from four residues 5 through 8 for compstatin. The classification of $\beta-$ turns is determined according to the criteria of Wilmot and Thornton [23]. The conformational space of $\beta$-turns is divided into four regions, $\alpha_{R}, \beta, \alpha_{L} / \gamma$, and $\varepsilon$, using the backbone dihedral angles $(\phi, \varphi)$ of central two residues 6 and 7 of the turns [23]. Table 1 shows the dihedral angles of $\beta-$ turns for two central residues Asp ${ }^{6}-\operatorname{Trp}^{7}$. Additional criteria for the $\beta$-turn formation are as follows: the distance between

Table 1

Dihedral angles of $\beta$-turns ${ }^{\mathrm{a}}$ for two central residues Asp $^{6}$-Trp ${ }^{7}$

\begin{tabular}{ll}
\hline Turn type & Dihedral angles \\
\hline$\alpha_{\mathrm{R}}$ & $\phi \leq 0^{\circ},-120^{\circ} \leq \varphi \leq 60^{\circ}$ \\
$\beta$ & $\phi \leq 0^{\circ}, \varphi>60^{\circ}$ or $\varphi<-120^{\circ}$ \\
$\alpha_{\mathrm{L}} / \gamma$ & $\phi>0^{\circ},-70^{\circ}<\varphi<110^{\circ}$ \\
$\varepsilon$ & $\phi>0^{\circ}, \varphi \geq 110^{\circ}$ or $\varphi \leq-70^{\circ}$ \\
\hline
\end{tabular}

${ }^{a}$ The criteria of $\beta$-turn formation are as follows: the distance between $\alpha$-carbons of $\mathrm{Gln}^{5}$ and $\mathrm{Gly}^{8}$ is less than 8.50 and the central turn residues Asp $^{6}-\operatorname{Trp}^{7}$ are not helical.

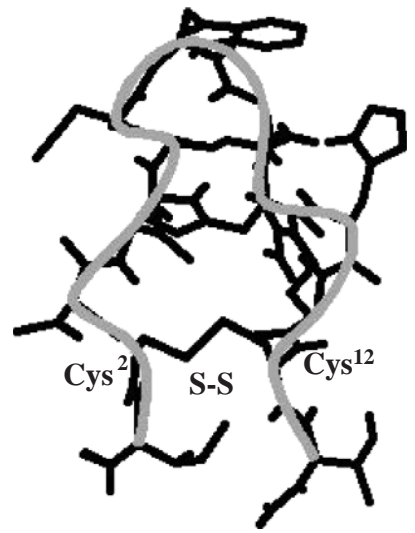

Fig. 2. The structure of < compstatin $>_{\text {ave }}$ obtained after the local minimization of the average structure of 21 NMR structures. Two residues $\mathrm{Cys}^{2}$ and $\mathrm{Cys}^{12}$ are bridged by a disulfide bond. The $<$ compstatin $>_{\text {ave }}$ has the coil $/ \alpha_{R}-\alpha_{R}$ conformation.

$\alpha$-carbon atoms of residues 5 and $8, \mathrm{Gln}^{5}-\mathrm{Gly}^{8}$, is less than 8.50 and the central two residues $\mathrm{Asp}^{6}-\operatorname{Trp}^{7}$ are not helical.

The average structure is obtained by averaging the coordinates of the 21 NMR structures. The local minimization of the average structure is carried out, and the minimized structure is represented as <compstatin $>_{\text {ave }}$ which is a coil $/ \alpha_{R}-\alpha_{R}$ conformation, as shown in Fig. 2.

Table 2

The locally minimized NMR structures of compstatin

\begin{tabular}{|c|c|c|c|}
\hline Structure & $E(\mathrm{kcal} / \mathrm{mol})$ & Conformation $^{\mathrm{a}}$ & $\operatorname{rmsd}^{\mathrm{b}}(\AA)$ \\
\hline$<$ compstatin $>_{1}$ & -482.84 & $\operatorname{Coil} / \alpha_{R}-\alpha_{R}$ & 2.61 \\
\hline$<$ compstatin $>_{2}$ & -479.37 & $\begin{array}{l}\beta \text {-hairpin }(3-4,10-11) / \\
\alpha_{R}-\alpha_{R}{ }^{c}\end{array}$ & 2.77 \\
\hline$<$ compstatin $>_{3}$ & -466.72 & $\operatorname{Coil} / \alpha_{R}-\alpha_{R}$ & 2.88 \\
\hline$<$ compstatin $>_{4}$ & -470.71 & $\operatorname{Coil} / \alpha_{R}-\alpha_{R}$ & 1.90 \\
\hline$<$ compstatin $>_{5}$ & -453.71 & $\operatorname{Coil} / \alpha_{R}-\alpha_{R}$ & 2.90 \\
\hline$<$ compstatin $>_{6}$ & -481.53 & Coil $/ \alpha_{R}-\alpha_{R}$ & 2.92 \\
\hline$<$ compstatin $>_{7}$ & -481.48 & $\operatorname{Coil} / \alpha_{R}-\alpha_{R}$ & 2.84 \\
\hline$<$ compstatin $>_{8}$ & -472.78 & $\operatorname{Coil} / \alpha_{R}-\alpha_{R}$ & 2.96 \\
\hline$<$ compstatin $>_{9}$ & -481.14 & $\operatorname{Coil} / \alpha_{R}-\alpha_{R}$ & 2.76 \\
\hline$<$ compstatin $>_{10}$ & -467.39 & Coil $/ \alpha_{R}-\alpha_{R}$ & 2.47 \\
\hline$<$ compstatin $>_{11}$ & -473.91 & $\operatorname{Coil} / \alpha_{R}-\alpha_{R}$ & 1.47 \\
\hline$<$ compstatin $>_{12}$ & -477.55 & $\operatorname{Coil} / \alpha_{R}-\alpha_{R}$ & 3.06 \\
\hline$<$ compstatin $>_{13}$ & -477.48 & $\begin{array}{l}\beta \text {-hairpin }(2-3,10-11) / \\
310 \text {-helix }(6-8)^{\mathrm{d}}\end{array}$ & 3.29 \\
\hline$<$ compstatin $>_{14}$ & -478.20 & $\operatorname{Coil} / \alpha_{R}-\alpha_{R}$ & 2.40 \\
\hline$<$ compstatin $>_{15}$ & -475.27 & $\operatorname{Coil} / \alpha_{R}-\alpha_{R}$ & 2.04 \\
\hline$<$ compstatin $>_{16}$ & -466.63 & $\operatorname{Coil} / \alpha_{R}-\alpha_{R}$ & 1.90 \\
\hline$<$ compstatin $>_{17}$ & -466.15 & Coil $/ \alpha_{R}-\alpha_{R}$ & 2.99 \\
\hline$<$ compstatin $>_{18}$ & -467.23 & $3_{10}$-helix $(6-8)$ & 2.85 \\
\hline$<$ compstatin $>_{19}$ & -480.47 & $\operatorname{Coil} / \alpha_{R}-\alpha_{R}$ & 3.35 \\
\hline$<$ compstatin $>_{20}$ & -467.57 & $3_{10}$-helix(6-8) & 3.23 \\
\hline$<$ compstatin $>_{21}$ & -472.73 & $\operatorname{Coil} / \alpha_{R}-\alpha_{R}$ & 3.10 \\
\hline$<$ compstatin $>_{\text {ave }}$ & -470.96 & Coil $/ \alpha_{R}-\alpha_{R}$ & - \\
\hline
\end{tabular}

a The secondary structure and the type of $\beta$-turn are represented before and after the slash, respectively.

${ }^{b}$ rmsd values using backbone atoms between $<$ compstatin $>_{\text {ave }}$ and $<$ compstatin $>_{i}(i=1-21)$ structures.

c $\beta$-hairpin $(3-4,10-11) / \alpha_{R}-\alpha_{R}$ represents $\beta$ structures between residues 3-4 and 10-11 with the $\alpha_{R}-\alpha_{R}$ turn type for two central residues, Asp ${ }^{6}-\operatorname{Trp}^{7}$.

d $\beta$-hairpin(2-3,10-11)/3 10 -helix(6-8) represents $\beta$ structures between residues $2-3$ and $10-11$ with a 310 -helix spanning residues 6-7-8. 


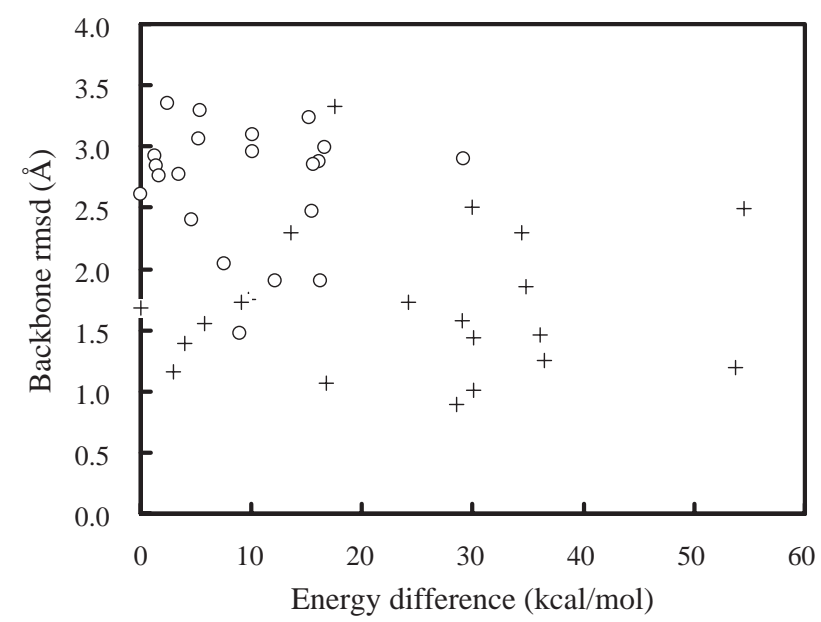

Fig. 3. The backbone rmsd versus energy for the 21 minimized NMR structures. Our result is represented as open circles and that of Klepeis et al. (from Ref. [15]) is represented as crosses. In Ref. [15], Klepeis et al. used $\mathrm{ECEFF} / 3$ potential as well as the restraints from the NMR data, and the calculated minimized energies are ranged from -17.12 to $-71.61 \mathrm{kcal} / \mathrm{mol}$. For comparison, energies are shifted by $71.61 \mathrm{kcal} / \mathrm{mol}$, so that the lowest energy corresponds to zero.

The backbone rmsd between the average structure and its minimized structure is 2.40 . Table 2 shows the locally minimized 21 NMR structures of compstatin, designated as $<$ compstatin $>_{i}(i=1-21)$. The original 21 PDB structures are all in coil conformations. However, after minimization, a $\beta$ hairpin $\left(<\right.$ compstatin $\left.>_{2}\right)$ as well as $3_{10}$-helix structures $\left(<\right.$ compstatin $>_{18}$ and $<$ compstatin $\left.>_{20}\right)$ [17] appeared (see the third column in Table 2). $3_{10}$-Helices are formed in the turn position with a relatively high energy. A $\beta$-hairpin structure with a $31_{10}$-helix in the turn position is also observed $\left(<\right.$ compstatin $\left.>{ }_{13}\right)$. It means that a $\beta$-turn can be converted to a 310 -helix. The backbone rmsd values between $<$ compstatin $>_{\text {ave }}$ and $<$ compstatin $>_{i}(i=1 \sim 21)$ range from 1.47 to 3.35 . The average rmsd and the standard deviation of the $21 \mathrm{rmsd}$ values are 2.70 and 0.50 , respectively. All structures with rmsd values less than 2.0 are in coil $/ \alpha_{R}-\alpha_{R}$ conformations. Fig. 3 shows the plot of rmsd versus energy for the 21 minimized structures. The lowest energy, $-482.84 \mathrm{kcal} / \mathrm{mol}$, is shifted to zero $\mathrm{kcal} / \mathrm{mol}$, and so the energy difference is represented on the $x$-axis. The results of Klepeis et al. [24], which were obtained using ECEFF/3 potential, are also shown for comparison. They calculated compstatin structure using the conformational restraints from NMR data. The calculated ECEFF/3 energies range from -17.12 to $-71.61 \mathrm{kcal} / \mathrm{mol}$. The lowest ECEFF $/ 3$ energy is also shifted to zero $\mathrm{kcal} / \mathrm{mol}$. As shown in Fig. 3, the rmsd values are distributed over rather wide ranges for both results. It implies that the 13 residue compstatin structure is rather unstable in solution. The energy distribution of our result is narrower than that of Klepeis et al. [24]. It should be noted that the conformations in this work are generated without using restraints from NMR data.

Table 3 lists the structures corresponding to the lowest energy and the least rmsd from twenty CSA runs. The backbone rmsd is calculated between $<$ compstatin $>_{\text {ave }}$ and structures obtained by CSA. Among the lowest-energy structures, conformations containing an $\alpha$-helix are found in two runs, $\mathrm{r} 12$ and $\mathrm{r} 19$. A $\beta$-hairpin containing structure is also found in run r16. However, most of the lowest-energy structures are in the coil with a $\beta$-turn state. Now, we examine the structures with the least rmsd. The average and the standard deviation of the rmsd values are 2.51 and 0.40 ,

Table 3

The structures with the lowest energy and with the least rmsd for 20 CSA runs

\begin{tabular}{|c|c|c|c|c|c|c|}
\hline & $E_{\text {lowest }}{ }^{\mathrm{a}}(\mathrm{kcal} / \mathrm{mol})$ & Conformation $^{\mathrm{a}}$ & $\operatorname{rmsd}(\AA)^{\mathrm{a}}$ & $E_{\text {rmsd }} \mathrm{b}(\mathrm{kcal} / \mathrm{mol})$ & Conformation $^{\mathrm{b}}$ & $\operatorname{rmsd}(\AA)^{b}$ \\
\hline $\mathrm{r} 1$ & -491.19 & $\operatorname{Coil} / \alpha_{R}-\alpha_{R}$ & 4.22 & -473.01 & Coil $/ \beta-\beta$ & 2.30 \\
\hline $\mathrm{r} 2$ & -491.53 & Coil/no- $\beta-$ turn $^{\mathrm{c}}$ & 4.37 & -448.00 & $\operatorname{Coil} / \varepsilon-\alpha_{R}$ & 2.52 \\
\hline $\mathrm{r} 3$ & -490.86 & $\operatorname{Coil} / \beta-\alpha_{L} / \gamma$ & 3.73 & -470.73 & $\operatorname{Coil} / \varepsilon-\alpha_{R}$ & 2.22 \\
\hline $\mathrm{r} 4$ & -490.46 & Coil/no- $\beta$-turn & 2.90 & -440.06 & $\operatorname{Coil} / \varepsilon-\alpha_{R}$ & 1.78 \\
\hline r5 & -490.17 & Coil $/ \beta-\alpha_{R}$ & 3.88 & -459.90 & Coil $/ \alpha_{R}-\beta$ & 2.65 \\
\hline r6 & -493.22 & $\operatorname{Coil} / \varepsilon-\alpha_{R}$ & 4.20 & -462.71 & $\operatorname{Coil} / \alpha_{R}-\alpha_{R}$ & 2.37 \\
\hline $\mathrm{r} 7$ & -491.89 & Coil $/ \alpha_{R}-\alpha_{R}$ & 4.76 & -455.21 & Coil/no- $\beta$-turn & 2.71 \\
\hline r8 & -490.17 & Coil $/ \alpha_{R}-\alpha_{R}$ & 4.05 & -470.04 & Coil $/ \alpha_{R}-\varepsilon$ & 2.71 \\
\hline r9 & -491.51 & Coil/no- $\beta$-turn & 4.37 & -456.30 & Coil $/ \alpha_{R}-\beta$ & 2.52 \\
\hline $\mathrm{r} 10$ & -492.49 & Coil $/ \beta-\beta$ & 4.85 & -462.69 & Coil $/ \alpha_{R}-\alpha_{R}$ & 3.08 \\
\hline r11 & -490.62 & $\operatorname{Coil} / \alpha_{R}-\beta$ & 4.01 & -453.98 & Coil $/ \alpha_{R}-\alpha_{R}$ & 1.77 \\
\hline $\mathrm{r} 12$ & -490.32 & $\alpha-\operatorname{helix}(3-6)$ & 3.23 & -465.53 & $\operatorname{Coil} / \varepsilon-\beta$ & 2.61 \\
\hline $\mathrm{r} 13$ & -491.05 & $\operatorname{Coil} / \beta-\alpha_{R}$ & 3.77 & -456.56 & Coil $/ \beta-\alpha_{R}$ & 2.60 \\
\hline r14 & -490.05 & Coil/no- $\beta$-turn & 3.73 & -454.59 & Coil/no- $\beta$-turn & 3.01 \\
\hline $\mathrm{r} 15$ & -490.50 & Coil/no- $\beta$-turn & 4.47 & -481.00 & Coil/no- $\beta$-turn & 3.01 \\
\hline r16 & -490.95 & $\beta$-hairpin $(1-2,12-13) / \alpha_{R}-\alpha_{R}$ & 3.37 & -463.45 & $\operatorname{Coil} / \varepsilon-\alpha_{L} / \gamma$ & 2.79 \\
\hline r17 & -490.59 & $\operatorname{Coil} / \alpha_{R}-\alpha_{R}$ & 4.36 & -463.89 & $\operatorname{Coil} / \alpha_{R}-\beta$ & 2.42 \\
\hline $\mathrm{r} 18$ & -490.18 & Coil $/ \alpha_{R}-\alpha_{R}$ & 3.13 & -482.87 & $\beta-$ hairpin $(3-5,9-10) / \alpha_{R}-\beta$ & 1.67 \\
\hline r19 & -490.41 & $\alpha-\operatorname{helix}(4-7)$ & 3.82 & -453.69 & Coil/no- $\beta$-turn & 2.91 \\
\hline $\mathrm{r} 20$ & -490.36 & Coil/no- $\beta$-turn & 5.11 & -456.71 & $\operatorname{Coil} / \alpha_{R}-\alpha_{L} / \gamma$ & 2.64 \\
\hline
\end{tabular}

${ }^{a}$ Energy, conformation and rmsd value of the structure with the lowest energy.

b Energy, conformation and rmsd value of the structure with the least rmsd value.

${ }^{c}$ No- $\beta$-turn means that the corresponding conformations do not satisfy the $\beta$-turn criterion. 
Table 4

Classification of the 1000 structures obtained from 20 independent CSA runs

\begin{tabular}{|c|c|c|c|}
\hline Family of structures & Population & $E_{\text {ave }}(\mathrm{kcal} / \mathrm{mol})$ & Average rmsd $(\AA)$ \\
\hline 1. Coil/no- $\beta$-turn & 476 & $-463.35( \pm 12.47)$ & $4.01( \pm 0.69)$ \\
\hline 2. Coil $/ \alpha_{R}-\alpha_{R}$ & 153 & $-466.34( \pm 11.11)$ & $3.84( \pm 0.77)$ \\
\hline 3. Coil $/ \alpha_{R}-\beta$ & 72 & $-465.53( \pm 11.19)$ & $3.82( \pm 0.66)$ \\
\hline 4. $\operatorname{Coil} / \varepsilon-\alpha_{\mathrm{R}}$ & 63 & $-465.94( \pm 14.96)$ & $3.85( \pm 0.77)$ \\
\hline 5. Coil $/ \beta-\alpha_{R}$ & 61 & $-468.49( \pm 11.58)$ & $3.86( \pm 0.54)$ \\
\hline 6. Coil $/ \beta-\alpha_{L} / \gamma$ & 35 & $-460.80( \pm 17.27)$ & $3.73( \pm 0.80)$ \\
\hline 7. Coil $/ \beta-\beta$ & 26 & $-474.03( \pm 9.83)$ & $3.74( \pm 0.75)$ \\
\hline 8. Coil $/ \varepsilon-\beta$ & 18 & $-468.80( \pm 10.06)$ & $4.18( \pm 0.65)$ \\
\hline 9. Coil $/ \alpha_{L} / \gamma-\alpha_{L} / \gamma$ & 15 & $-462.10( \pm 10.79)$ & $3.90( \pm 0.83)$ \\
\hline 10. Coil $/ \alpha_{L} / \gamma-\alpha_{R}$ & 13 & $-465.36( \pm 15.01)$ & $4.08( \pm 0.83)$ \\
\hline 11. Coil $/ \beta-\varepsilon$ & 3 & $-453.87( \pm 14.15)$ & $3.79( \pm 1.11)$ \\
\hline 12. Coil $/ \alpha_{L} / \gamma-\beta$ & 3 & $-447.66( \pm 24.03)$ & $3.71( \pm 0.83)$ \\
\hline 13. Coil $/ \alpha_{R}-\varepsilon$ & 2 & $-471.51( \pm 2.09)$ & $3.77( \pm 1.49)$ \\
\hline 14. Coil $/ \varepsilon-\alpha_{L} / \gamma$ & 2 & $-460.75( \pm 3.81)$ & $2.92( \pm 0.19)$ \\
\hline 15. Coil $/ \alpha_{R}-\alpha_{L} / \gamma$ & 1 & -456.71 & 2.64 \\
\hline 16. Coil $/ \alpha_{L} / \gamma-\varepsilon$ & 1 & -464.45 & 4.50 \\
\hline 17. $3_{10}-$ helix $(2-4) / \alpha_{R}-\alpha_{R}$ & 10 & $-473.08( \pm 9.05)$ & $3.35( \pm 0.33)$ \\
\hline 18. 310 -helix $(2-4) / \beta-\alpha_{L} / \gamma$ & 1 & -455.43 & 5.70 \\
\hline 19. $3_{10}$-helix $(2-4) /$ no- $\beta$-turn & 2 & $-462.89( \pm 11.62)$ & $4.71( \pm 0.25)$ \\
\hline 20. $3_{10}$-helix $(10-12) /$ no- $\beta$-turn & 2 & $-454.52( \pm 0.94)$ & $4.04( \pm 0.69)$ \\
\hline 21. 310-helix (6-9) & 10 & $-467.59( \pm 9.55)$ & $3.95( \pm 0.48)$ \\
\hline 22. 310-helix (7-9) & 6 & $-471.12( \pm 9.03)$ & $3.62( \pm 0.36)$ \\
\hline 23. $3_{10}$-helix $(8-10)$ & 7 & $-465.01( \pm 10.42)$ & $3.94( \pm 0.23)$ \\
\hline 24. 310 -helix $(6-8)$ & 4 & $-462.86( \pm 10.46)$ & $3.98( \pm 0.15)$ \\
\hline 25. 310 -helix $(3-5)$ & 2 & $-467.80( \pm 13.98)$ & $4.10( \pm 0.54)$ \\
\hline 26. $\pi$-helix $(2-6)$ & 2 & $-468.21( \pm 19.11)$ & $3.75( \pm 0.11)$ \\
\hline 27. $\beta$-hairpin $/ \alpha_{R}-\alpha_{R}$ & 2 & $-484.60( \pm 8.99)$ & $3.31( \pm 0.09)$ \\
\hline 28. $\beta$-hairpin $/ \alpha_{R}-\beta$ & 1 & -482.87 & 1.67 \\
\hline 29. $\alpha$-helix (3-6) & 5 & $-475.15( \pm 14.90)$ & $3.48( \pm 0.45)$ \\
\hline 30. $\alpha$-helix (4-7) & 1 & -490.41 & 3.82 \\
\hline 31. $\alpha$-helix (6-9) & 1 & -455.44 & 3.74 \\
\hline
\end{tabular}

respectively. The structures with the least rmsds below 2.00 are $\operatorname{coil} / \varepsilon-\alpha_{R}$, coil $/ \alpha_{R}-\alpha_{R}$, and $\beta$-hairpin $/ \alpha_{R}-\beta$ in runs $r 4$, r11 and r18, respectively. This result demonstrates that CSA found conformations very similar to the experimentally observed structures (coil $\left./ \alpha_{R}-\alpha_{R}\right)$ as well as many other local minimum-energy conformations.

Table 4 summarizes the 1000 structures of compstatin, obtained from the $20 \mathrm{CSA}$ runs, which are classified into (a)

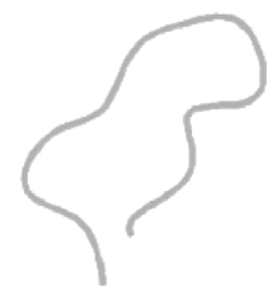

(d)

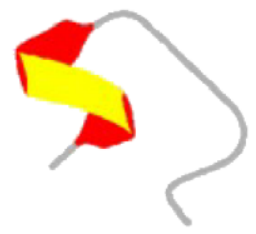

(b)

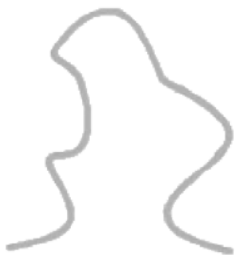

(e)

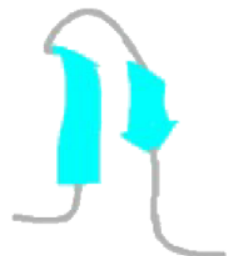

(c)

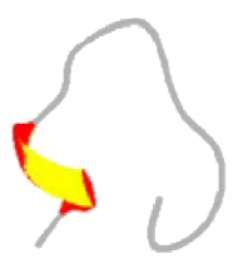

(f)

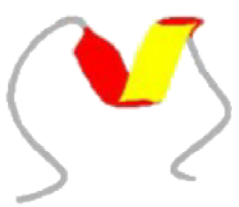

Fig. 4. Six representative conformations of (a) coil/no- $\beta$-turn, (b) coil $/ \alpha_{R}-\alpha_{R}$, (c) 310 -helix (2-4)/ $\alpha_{R}-\alpha_{R}$, (d) $\pi$-helix (2-6), (e) $\beta$-hairpin $/ \alpha_{R}-\beta$, and (f) $\alpha$-helix (6-9) 


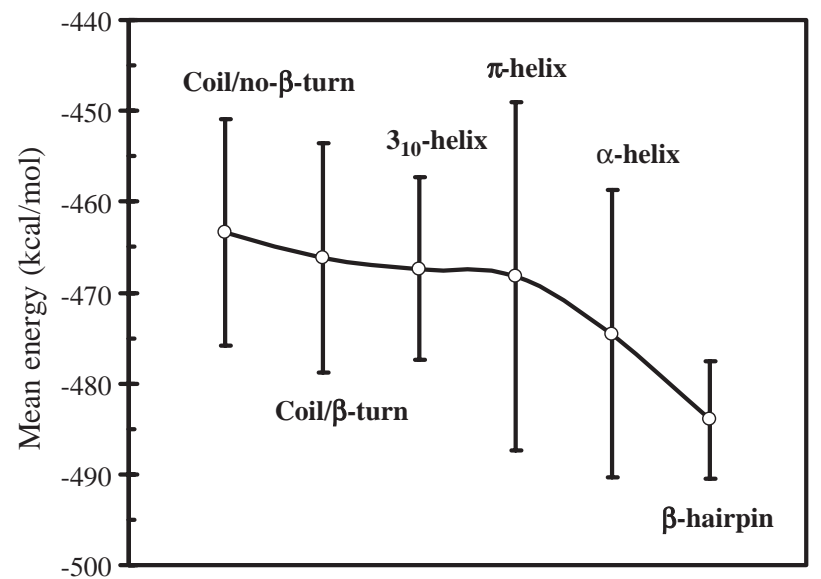

Six representative conformations of compstatin structures

Fig. 5. The average energies of the six representative conformations from thirty-one compstatin families are shown. They are coil/no- $\beta$-turn, coil with $\beta$-turn, $3_{10}$-helix, $\pi$-helix, $\beta$-hairpin, and $\alpha$-helix. Twenty independent CSA runs starting from random states of compstatin have generated 1000 structures, which are classified into thirty-one families. Among these 1000 structures, the population of coil without $\beta$-turns is $47.6 \%$, coil with a $\beta$ turn is $46.8 \%$, a 310 -helix is $4.4 \%$, an $\alpha$-helix is $0.7 \%$, a $\beta$-hairpin is $0.3 \%$, and a $\pi$-helix is $0.2 \%$. The error bars correspond to the standard deviation.

thirty-one structural families. The number of population, the average values of energy and rmsd for these 31 families of compstatin structures are given in the table. Among the 1000 structures, the population of coil without a $\beta$-turn is $47.6 \%$, coil with a $\beta$-turn is $46.8 \%, 3_{10}$-helix is $4.4 \%, \alpha$ helix is $0.7 \%$, $\beta$-hairpin is $0.3 \%$, and $\pi$-helix is $0.2 \%$. Fig. 4 shows six representative conformations having coil/no- $\beta$ turn, coil $/ \alpha_{R}-\alpha_{R}, 3_{10}$-helix, $\pi$-helix, $\beta$-hairpin, and $\alpha$-helix.

The conformational space of a $\beta$-turn is divided into four regions, $\alpha_{R}, \beta, \alpha_{L} / \gamma$, and $\varepsilon$, for each residue of two central residues 6 and 7 of the turn. Thus, there may exist a total of sixteen types of $\beta$-turns. Among the 468 coil structures with a $\beta$-turn, the major conformation is coil $/ \alpha_{R}-\alpha_{R}$. Coil conformations with $\alpha_{R}-\alpha_{R}, \alpha_{R}-\beta, \varepsilon-\alpha_{R}, \beta-\alpha_{R}, \beta-\alpha_{L} / \gamma, \beta-\beta, \varepsilon-$ $\beta, \alpha_{L} / \gamma-\alpha_{L} / \gamma$, and $\alpha_{L} / \gamma-\alpha_{R}$ turn types are found with a probability greater than $1 \%$. However, families 11 to 16 having coil conformations with $\beta-\varepsilon, \alpha_{L} / \gamma-\beta, \alpha_{R}-\varepsilon, \varepsilon-\alpha_{L} / \gamma$, $\alpha_{R}-\alpha_{L} / \gamma$, and $\alpha_{L} / \gamma-\varepsilon$ turn types are found with a very low probability. Interestingly, among the possible 16 turn types, coil conformation with $\varepsilon-\varepsilon$ turn type has not been found.

Families $17-25$ contain a 310 -helix. The 310 -helix is formed in residues spanning $2-4,10-12,6-9,7-9,8-10,6-$

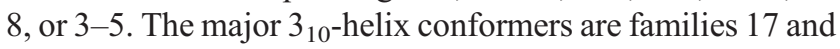

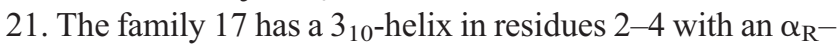
$\alpha_{R}$ turn. It is interesting to observe that the family 21 has a $3_{10}$-helix over residues 6-9 in the turn region. The family 31 has an $\alpha$-helix over residues $6-9$. From these results, it can be concluded that the turn region (residues 5-8) can be converted, although rarely, to a $3_{10}$-helix and an $\alpha$-helix.

The average energy of coil conformers without any type of $\beta$-turn is $-465.35( \pm 12.47) \mathrm{kcal} / \mathrm{mol}$, as shown in Table 4. All the conformers including coil with a $\beta$-turn, $3_{10}$-helix, $\pi$-helix, $\beta$-hairpin, and $\alpha$-helix have lower energies than coil/no- $\beta$-turn with their average energies, -466.16 $( \pm 12.67),-467.40 \quad( \pm 10.03),-468.21 \quad( \pm 19.11)$, $-484.02( \pm 6.43)$, and $-474.52( \pm 15.84) \mathrm{kcal} / \mathrm{mol}$, respectively (see Fig. 5). It means that conformations with welldefined secondary structures are more stable than coil conformations within the energy range of the restricted CSA search. Among coil conformations, coil with a $\beta$-turn is found to be more stable than coil without a $\beta$-turn.

\section{Conclusions}

We have applied the CSA method to the 13-residue complement inhibitor peptide, compstatin, using an all-atom force field and a continuum solvent model, and we have identified the structural characteristics of the compstatin molecule in solution according to the energetics dictated by the combined potential. In order to sample conformations in the energy range of the minimized NMR structures, we have used the stopping criterion to terminate the CSA search when a conformation whose energy is less than $-490 \mathrm{kcal} /$ mol is found. If we do not use this stopping criterion and continue the CSA search, very low energy conformations with energy of $-520 \mathrm{kcal} / \mathrm{mol}$ are retained in the bank. However, these low energy conformations are almost all in coil states without $\beta$-turns. When the stopping criterion is used, a great variety of conformations are generated around experimentally known structures. Twenty independent CSA runs starting from random states have found 1000 representative conformations in the energy landscape of compstatin, which are classified into thirty-one structural families. Total population of coil structures is $94.4 \%$. Other conformers containing a $3{ }_{10}$-helix, a $\pi$-helix, a $\beta$-hairpin, and an $\alpha$-helix are also found. Coil/no- $\beta$-turn has higher energy than the other conformations.

\section{References}

[1] J.D. Lambris, V.M. Holers (Eds.), Therapeutic Interventions in the Complement System, Humana Press, Totowa, NJ, 2000.

[2] H.J. Muller-Eberhard, Complement-chemistry and pathways, in: J.I. Gallin, I.M. Goldstein, R. Synderman (Eds.), Inflammation: Basic Principles and Clinical Correlates, 2nd ed., Raven Press, New York, 1992.

[3] M.M. Frank, L.F. Fries, The role of complement in inflammation and phagocytosis, Immunol. Today 12 (1991) 322-326.

[4] K.R. Kalli, P. Hsu, D.T. Fearon, Therapeutic uses of recombinant complement protein inhibitors, Springer Semin. Immunopathol. 15 (1994) 417-431.

[5] U.S. Vasthare, R.H. Rosenwasser, F.C. Barone, R.F. Tuma, Involvement of the complement system in cerebral ischemic and reperfusion injury, FASEB J. 7 (1993) A424.

[6] B.M. Bradt, W.P. Kolb, N.R. Cooper, Complement-dependent proinflammatory properties of the Alzheimer's disease $\beta$-peptide, J. Exp. Med. 188 (1998) 431-438.

[7] J. Rogers, N.R. Cooper, S. Webster, J. Schultz, P.L. McGeer, S.D. Styren, W.H. Civin, L. Brachova, B. Bradt, P. Ward, I. Lieberburg, 
Complement activation by $\beta$-amyloid in Alzheimer-disease, Proc. Natl. Acad. Sci. 89 (1992) 10016-10020.

[8] R.A. Robbins, W.D. Russ, J.K. Rasmussen, M.M. Clayton, Activation of the complement system in the adult respiratory distress syndrome, Am. Rev. Respir. Dis. 135 (1987) 651-658.

[9] K.S. Kilgore, G.S. Friedrichs, J.W. Homeister, B.R. Lucchesi, The complement system in myocardial ischaemia/reperfusion injury, Cardiovasc. Res. 28 (1994) 437-444.

[10] R. Gallinaro, W.G. Cheadle, K. Applegate, H.C. Polk Jr., The role of the complement system in trauma and infection, Surg. Gynecol. Obstet. 174 (1992) 435-440.

[11] A. Sahu, D. Morikis, J.D. Lambris, Complement inhibitors targeting C3, C4, and C5, in: J.D. Lambris, V.M. Holers (Eds.), Therapeutic Interventions in the Complement System, Humana Press, Totowa, NJ, 2000, pp. 75-112.

[12] A. Sahu, B.K. Kay, J.D. Lambris, Inhibition of human complement by a C3-binding peptide isolated from a phage-displayed random peptide library, J. Immunol. 157 (1996) 884-891.

[13] A. Sahu, A.M. Soulika, D. Morikis, L. Spruce, W.T. Moore, J.D. Lambris, Binding kinetics, structure-activity relationship, and biotransformation of the complement inhibitor compstatin, J. Immunol. 165 (2000) 2491-2499.

[14] D. Morikis, N. Assa-Munt, A. Sahu, J.D. Lambris, Solution structure of compstatin, a potent complement inhibitor, Protein Sci. 7 (1998) 619-627.

[15] D. Wales, Energy Landscapes: Applications to Clusters, Biomolecules and Glasses, Cambridge University Press, New York, 2004.
[16] J.N. Onuchic, Z. Luthey-Schulten, P.G. Wolynes, Theory of protein folding: the energy landscape perspective, Annu. Rev. Phys. Chem. 48 (1997) 545-600.

[17] B. Mallik, J.D. Lambris, D. Morikis, Conformational interconversion in compstatin probed with molecular dynamics simulations, Proteins 52 (2003) $130-141$.

[18] S.-Y. Kim, S.J. Lee, J. Lee, Conformational space annealing and an off-lattice frustrated model protein, J. Chem. Phys. 119 (2003) $10274-10279$.

[19] S.-Y. Kim, S.J. Lee, J. Lee, The energy landscape of a BLN protein with beta-hairpin shape, J. Korean Phys. Soc. 44 (2004) 589-593.

[20] B.V.R. Brooks, R.E. Bruccoleri, B.D. Olafson, D.J. States, S. Swaminathan, M. Karplus, CHARMM: a program for macromolecular energy, minimization and dynamics calculations, J. Comput. Chem. 4 (1983) 187-217.

[21] W. Kabsch, C. Sander, Dictionary of protein secondary structure: pattern recognition of hydrogen-bonded and geometrical features, Biopolymers 22 (1983) 2577-2637.

[22] R. Koradi, M. Billeter, K. Wuthrich, MOLMOL: a program for display and analysis of macromolecular structures, J. Mol. Graph. 14 (29-32) (1996) 51-55.

[23] C.M. Wilmot, J.M. Thornton, Beta-turns and their distortions: a proposed new nomenclature, Protein Eng. 3 (1990) 479-493.

[24] J.L. Klepeis, C.A. Floudas, D. Morikis, J.D. Lambris, Predicting peptide structures using NMR data and deterministic global optimization, J. Comput. Chem. 20 (1999) 1354-1370. 